\title{
D'une scène à l'autre, la concertation comme itinéraire
}

\author{
Jean-Eudes Beuret ${ }^{\mathrm{a}}$, Stéphane Pennanguer ${ }^{\mathrm{b}}$, Fanny Tartarin ${ }^{\mathrm{b}}$ \\ a Agroéconomiste, Département Économie rurale et gestion, Agrocampus Rennes, 65 rue de Saint-Brieuc, CS 84215, \\ 35042 Rennes cedex, France \\ b Halieute, Département halieutique, Agrocampus Rennes, 65 rue de Saint-Brieuc, CS 84215, 35042 Rennes cedex, France
}

\begin{abstract}
Les auteurs défendent la thèse selon laquelle la concertation se développe le plus souvent hors des procédures qui lui sont initialement dédiées. Pour cela, ils s'appuient sur des exemples diversifiés d'expériences de gestion de biens environnementaux soumis à des usages concurrents. Ils en concluent que la concertation déborde constamment des formes instituées et qu'il importe, pour l'accompagner, d'en identifier les contours et configurations évolutifs, rarement définis à l'avance. Le recours original à la métaphore de la scène, probablement à rapprocher du concept d'arène utilisé par certains politologues et anthropologues, offre des perspectives méthodologiques intéressantes pour la décrire et l'analyser.
\end{abstract}

La Rédaction

\section{Mots-clés :}

concertation ; itinéraire

de concertation ; réseaux d'acteurs; sociologie de l'innovation

\section{Keywords:}

concertation; concertation routes; stakeholder networks; sociology of innovation
Résumé - La gestion de biens d'environnement soumis à des usages concurrents suppose bien souvent une concertation associant usagers et gestionnaires. Les itinéraires de concertation représentent le cheminement effectivement suivi par la concertation : l'analyse comparative de divers itinéraires, en zone côtière, nous a permis d'identifier des facteurs qui conditionnent leur dynamique et de distinguer plusieurs morphotypes d'itinéraires. Nous constatons que la concertation ne se limite pas à des instances données, mais évolue d'une scène à une autre et se déplace de fait vers les scènes qui lui sont le plus favorables. Elle s'inscrit dans un champ constitué par des réseaux d'acteurs qui se construisent et se consolident via ces processus. Ceci nous conduit à mettre en cause une approche de la concertation comme un processus unifié, délimité en termes de thème, d'instances et de participants, puis à proposer des repères méthodologiques pour l'analyse, la représentation ou l'accompagnement de ces processus.

\begin{abstract}
Environmental management: the changing scenes of concertation routes. Management of environmental goods subject to rival uses requires concertation among users and public stakeholders. Concertation processes usually develop within procedures that set out the framework of the good to be managed. Studies of cooperation among stakeholders are usually restricted to the framework of the procedure provided for the concertation process. However, many concertation situations develop outside of these procedures. They follow their own routes within networks of stakeholders. We have defined the course followed by concertation processes within these networks as "concertation routes". A comparative analysis of these routes in a situation of conflicting uses in rural and coastal areas of Brittany shows that from an initial topic that triggers the process and involves a couple of stakeholders only, concertation moves on to other scenes, involving other stakeholders in other places on new topics. Concertation moves out of the provided procedures to the most favourable places, and develops in a wider field consisting of consolidating networks of stakeholders. Our analysis caused us to challenge the approach that considers concertation as a standardised process, defined by its topic and stakeholders, and to propose a methodology to represent and analyse these processes. It also shows the need for managers to accompany the concertation process rather than impose a too strict framework on it.
\end{abstract}

Auteur correspondant :

J.-E. Beuret, beuret@agrocampus-rennes.fr 


\section{Introduction}

Les espaces ruraux ou littoraux sont soumis à de multiples usages, parfois complémentaires, souvent concurrents. Face à cela, deux modes de gestion sont couramment employés (Mermet, 1992) : la gestion directe passe par l'acquisition des droits de propriété, le nouveau propriétaire pouvant gérer l'espace comme il l'entend, alors que la gestion indirecte revient à influencer ce que font les agents économiques par des réglementations, des incitations monétaires, etc. Mais ces outils ne permettent pas d'assurer la cohérence territoriale de l'action publique du fait de la dissémination de ces mesures dans l'espace et d'une fragmentation de la décision entre des acteurs qui n'agissent que sur un problème d'environnement, sans tenir compte des interdépendances existantes (Barouch, 1989). De plus, que faire dans des espaces restreints, tels que le golfe du Morbihan, où le milieu subit la montée en puissance de l'ostréiculture, des sports de plage, de la navigation de plaisance, de plusieurs types de pêche, de l'urbanisation ou du tourisme? Une concertation, tant entre les usagers qu'entre les intervenants publics, est nécessaire. Des procédures de concertation ont été mises en place et des textes, tels que la loi sur la démocratie de proximité, incitent les administrations à le faire. De fait, les dynamiques de concertation fleurissent à l'initiative d'administrations ou d'acteurs locaux, notamment en Bretagne (Beuret et Tréhet, 2001). Mais les promoteurs de ces dynamiques sont souvent désarmés lorsqu'il s'agit de savoir comment conduire un processus de concertation, car les pouvoirs publics disent qu'il faut le faire... mais pas comment le faire.

La plupart des travaux de recherche actuellement menés sur de tels processus portent sur des procédures mises en place autour d'un objet donné. Ce peut être un bien d'environnement (l'eau, un marais, un espace naturel sensible) ou un projet d'aménagement qui suscite un conflit. On s'intéresse à un ou des processus de concertation portés par une procédure ou une instance prévue à cet effet (SAGE, contrat de rivière, schéma de mise en valeur de la mer...) : le jeu d'acteurs étudié se trouve limité à ceux qui participent à la procédure ou à l'instance, qui s'y opposent ou qui la soutiennent. Pourtant, de nombreuses concertations se développent hors de toute procédure et, même lorsqu'il existe une «marche à suivre » dictée par le promoteur du processus, on s'aperçoit que ce ne sont que des points de passage obligés autour desquels le processus va suivre son propre chemin. Nous avons étudié douze de ces processus dans le cadre du projet CONCERT ${ }^{1}$, qui porte sur des dynamiques de concertation entre des agriculteurs et d'autres usagers des espaces ruraux en Bretagne, lequel nous a permis de

\footnotetext{
${ }^{1}$ Projet financé par le ministère de l'Écologie et du Développement durable dans le cadre du programme Concertation, Décision, Environnement.
}

distinguer ainsi plusieurs types d'«itinéraires de concertation ». Après avoir présenté la méthodologie employée pour l'analyse et la représentation de ces processus de concertation, nous caractériserons les différents types de processus observés, puis nous en tirerons les déductions opérationnelles et méthodologiques.

\section{Cadre conceptuel et méthodologie}

\section{Définitions et méthodologie d'analyse}

\section{De la concertation à la gestion concertée}

La concertation est l'un des termes qui semblent les plus flous dans les écrits des chercheurs comme dans les textes et les propositions des décideurs publics. Certains l'associent à une forme renouvelée de consultation, où le gouvernement conserve toute l'autorité décisionnelle (Lamoureux, 1996; Galléty et Rousset, 2000). La consultation n'offre aucune garantie quant à l'ouverture d'un dialogue entre les acteurs et celui qui consulte s'emploie parfois à éviter un débat qui donnerait un pouvoir aux acteurs locaux en leur permettant de se construire une vision commune : ceci constitue une différence majeure avec la concertation. D'autres auteurs associent la concertation à une négociation amputée de tout pouvoir décisionnel. Selon Mermet (1998), la concertation est moins avancée qu'une négociation, car elle ne cherche pas forcément une prise de décision d'un commun accord, le pouvoir de décision restant à l'initiative du maître d'ouvrage ou de l'administration. Mais, pour nous, la concertation va plus loin que la négociation sur d'autres plans, car, alors que la négociation vise à décider sans obligatoirement comprendre l'autre, la concertation vise à construire des objets communs essentiels pour l'avenir. Elle laisse par ailleurs les acteurs libres de construire ensemble la question posée. Finalement, ce qui fait l'intérêt de la concertation par rapport à la consultation ou à la négociation, c'est la construction collective d'objets qui vont devenir communs aux participants et sur la base desquels pourront émerger des initiatives, des décisions, des règles, à l'issue ou en aval de la concertation proprement dite. La concertation peut n'induire ni décision ni action collective, mais elle crée des références communes qui rendent possibles l'action et la décision collective.

Nous définissons la concertation comme un processus de construction collective de questions, de visions, d'objectifs et de projets communs relatifs à un objet. Dans la concertation :

- la question posée, la légitimité des acteurs admis à participer et les objectifs poursuivis par le processus ne sont pas donnés a priori, mais sont construits par le processus ; 
- le processus peut être « induit» par les pouvoirs publics ou se développer de façon autonome, entre des acteurs dont l'objectif est soit de se forger une position commune à défendre auprès des décideurs, soit d'agir ensemble indépendamment de la puissance publique ;

- la participation est volontaire, car l'individu est acteur du processus (alors qu'il peut subir une consultation de façon relativement passive ou être convoqué à une négociation);

- le décideur public, s'il est présent, peut décider de déléguer au collectif une partie de son pouvoir de décision, mais rien ne l'oblige à le faire. S'il le fait, ceci devient une règle qu'il devra respecter. Les règles du jeu vont être là aussi l'objet d'une construction collective.

La gestion concertée fait appel à la concertation pour dépasser les divergences de perceptions, d'intérêts et de positions en vue de construire une gestion cohérente et coordonnée d'un bien soumis à différents usages. Elle ouvre un espace doté des propriétés d'un espace public, qui sont la liberté et l'autonomie des citoyens pour la formation par la raison d'une opinion et d'une volonté collective (Habermas, 1978). Cet espace n'est pas toujours institutionnellement organisé, contrairement à la façon dont il est défini par Ladrière (1992), même si, en tant qu'espace public autonome, il est "garanti » par le système politique (Candau, 1999). Les concertations qui nous intéressent sont donc des espaces publics dotés d'objectifs particuliers, relatifs à la gestion coordonnée de biens communs.

\section{L'échantillon : entre diversité et comparabilité}

Sur la base de cette définition a été réalisé un inventaire régional (en Bretagne) de cas de concertation associant des agriculteurs et d'autres catégories d'acteurs, en vue d'une gestion concertée d'espaces ruraux ou littoraux. Certaines dynamiques de concertation ont été initiées par les pouvoirs publics dans des territoires où ils recherchent l'adhésion des acteurs locaux à leur démarche : elles sont qualifiées de descendantes, par opposition à des démarches ascendantes, plus informelles, issues d'initiatives locales, qui représentent les deux tiers des dynamiques identifiées. Les 86 cas identifiés ont été classés selon le caractère ascendant ou descendant de la dynamique, le degré de conflictualité puis d'institutionnalisation du processus (Beuret et Tréhet, 2001). La typologie nous a permis de sélectionner un échantillon où l'on retrouve la diversité des situations rencontrées sur le terrain. Mais, au sein de cette diversité, a été recherchée une certaine similarité de situations permettant des comparaisons pertinentes. L'échantillon est ainsi divisible en trois sous-groupes présentant des similarités (Tab. 1).
Nous ne mobiliserons que le premier sous-échantillon pour illustrer ici notre propos : il s'agit de concertations dont l'objet est la qualité de l'eau dans des rias par lesquelles la mer pénètre au sein de l'espace terrestre et constitue ainsi des lieux privilégiés pour l'ostréiculture, mais aussi le tourisme, le mouillage de bateaux de plaisance, l'observation des oiseaux, etc. À l'interface entre terre et mer, des activités ostréicoles sont menacées par des pollutions, en particulier d'origine agricole, ce qui engendre des conflits entre ostréiculteurs et agriculteurs. Dans la ria d'Étel, le conflit donne naissance à des espaces de dialogue entre professionnels qui vont se formaliser progressivement; en rivière de Penerf, la concertation progresse dans un contexte très conflictuel, sans instances formalisées, et dans l'Aven et le Belon, c'est une communauté de communes qui tente d'induire une concertation. Parallèlement et sur la base des processus qui ont émergé à Étel et Penerf, les deux professions ont mis en place une concertation entre leurs organes de représentation, à l'échelle d'un département. Nous reviendrons ponctuellement à d'autres cas étudiés, présentés dans le tableau 1.

\section{La méthodologie d'analyse des processus et ses limites}

L'analyse comparative repose sur l'application de la même grille d'analyse à tous les cas retenus. Les processus de concertation ont fait l'objet d'un suivi longitudinal : durant trois ans, nous avons rencontré périodiquement les participants et assisté à certains temps forts du débat. En amont de ce suivi a été réalisé un état des lieux initial, lors duquel nous avons reconstitué l'historique du processus, à travers la vision qu'en avaient les représentants des différentes catégories d'acteurs. L'analyse de chaque processus suppose de le construire en l'exhumant de la mémoire et du vécu des participants, via des entretiens semi-directifs complétés par la collecte de documents écrits (comptes rendus de réunions, articles de journaux, projets, etc.). Le nombre d'entretiens réalisés pour chaque cas, compris entre 3 et 28 , dépend de la diversité des points de vue des participants à la concertation.

Lors des entretiens, nous demandons à notre interlocuteur de nous raconter ce qui s'est passé «depuis le début » (il est intéressant de voir où il fixe ce début); puis, lorsqu'il évoque des moments-clés de la concertation ou des événements déterminants, nous l'amenons à préciser les ressorts de ces évolutions. Ces récits sont mis en parallèle afin de comparer l'interprétation faite par chacun d'événements similaires, puis nous construisons un historique détaillé précisant les divergences de vues relatives à telle ou telle étape. À partir de ce matériau de base, le processus est décomposé en étapes qui se succèdent ou se chevauchent. Puis nous recherchons, dans la logique de ce processus et en nous référant aux entretiens 
Tableau 1. L'échantillon CONCERT : entre diversité et comparabilité.

\begin{tabular}{|c|c|c|}
\hline $\begin{array}{l}\text { Un même } \\
\text { objet... }\end{array}$ & Des lieux & ... des processus différenciés \\
\hline \multirow{4}{*}{$\begin{array}{l}\text { L'objet : la } \\
\text { qualité de l'eau } \\
\text { dans des rias } \\
\text { soumises à des } \\
\text { usages agricoles } \\
\text { et ostréicoles }\end{array}$} & $\begin{array}{l}\text { Une rivière : la } \\
\text { ria d'Étel }\end{array}$ & $\begin{array}{l}\text { L'initiative de représentants professionnels locaux, qui se } \\
\text { formalise dans un contexte de dialogue }\end{array}$ \\
\hline & $\begin{array}{l}\text { Une rivière : la } \\
\text { rivière de Penerf }\end{array}$ & $\begin{array}{l}\text { Une initiative locale, dans un contexte de conflit, } \\
\text { qui reste informelle }\end{array}$ \\
\hline & $\begin{array}{l}\text { Des rivières: } \\
\text { l'Aven, le Belon }\end{array}$ & $\begin{array}{l}\text { L'initiative d'une communauté de communes pour un projet } \\
\text { d'intervention dans la gestion de ces biens d'environnement }\end{array}$ \\
\hline & $\begin{array}{l}\text { L'échelle du } \\
\text { département }\end{array}$ & $\begin{array}{l}\text { Une concertation interprofessionnelle agriculteurs- } \\
\text { ostréiculteurs pour une « charte de l'agriculture littorale » }\end{array}$ \\
\hline \multirow{3}{*}{$\begin{array}{l}\text { L'objet : de vastes } \\
\text { territoires } \\
\text { littoraux soumis à } \\
\text { des usages } \\
\text { concurrents et à } \\
\text { de multiples } \\
\text { controverses }\end{array}$} & $\begin{array}{l}\text { Un territoire : la } \\
\text { baie du Mont- } \\
\text { Saint-Michel }\end{array}$ & $\begin{array}{l}\text { Des scènes de concertation, mais pas de procédure unifiée à } \\
\text { l'échelle du territoire }\end{array}$ \\
\hline & $\begin{array}{l}\text { Un territoire : le } \\
\text { golfe du } \\
\text { Morbihan }\end{array}$ & $\begin{array}{l}\text { Une procédure ascendante sur l'espace terrestre (projet de parc } \\
\text { naturel régional). Une procédure descendante sur l'espace } \\
\text { marin (projet de schéma de mise en valeur de la mer) }\end{array}$ \\
\hline & $\begin{array}{l}\text { Un territoire : la } \\
\text { mer d'Iroise }\end{array}$ & $\begin{array}{l}\text { Une procédure d'initiative nationale (projet de parc national } \\
\text { marin), exogène au territoire }\end{array}$ \\
\hline \multirow{5}{*}{$\begin{array}{l}\text { L'objet : la } \\
\text { préservation de } \\
\text { l'eau et/ou du } \\
\text { paysage dans des } \\
\text { espaces ruraux } \\
\text { gérés de fait par } \\
\text { l'agriculture }\end{array}$} & $\begin{array}{l}\text { Une communauté } \\
\text { de communes }\end{array}$ & $\begin{array}{l}\text { Procédure de concertation proposée par le conseil général (35) } \\
\text { en vue d'une action collective contractualisée }\end{array}$ \\
\hline & $\begin{array}{l}\text { Un bassin } \\
\text { versant }\end{array}$ & $\begin{array}{l}\text { Démarche descendante visant à préserver la qualité de l'eau, } \\
\text { dans un bassin versant « Bretagne eau pure » }\end{array}$ \\
\hline & $\begin{array}{l}\text { Une communauté } \\
\text { d'agglomération }\end{array}$ & $\begin{array}{l}\text { Un cadre de concertation permanent entre élus et agriculteurs, } \\
\text { dans une communauté d'agglomération }\end{array}$ \\
\hline & Une commune & $\begin{array}{l}\text { L'initiative informelle d'une association locale en faveur du } \\
\text { paysage }\end{array}$ \\
\hline & Un département & $\begin{array}{l}\text { Une instance de concertation entre agriculteurs et } \\
\text { environnementalistes autour des aménagements fonciers }\end{array}$ \\
\hline
\end{tabular}

réalisés, les facteurs qui ont conditionné l'évolution de la concertation. L'analyse comparative permet d'identifier des facteurs qui reviennent de façon récurrente. Enfin, à l'issue de leur analyse, les dynamiques de concertation étudiées sont toutes représentées de la même façon, ce qui permet d'identifier des points communs, des différences et d'établir des comparaisons pertinentes.

Les limites de cette démarche tiennent à différents niveaux de subjectivité. C'est d'abord celle du discours des acteurs. Les divergences observées dans les discours nous intéressent, car elles résultent de vécus différents. Mais il arrive que l'interviewé modifie sciemment la réalité, car l'histoire est un enjeu important : se donner un rôle central dans le processus donne un surcroît de légitimité qui peut alimenter une position de force. Parfois, il a déjà admis comme vraie une version des faits qu'il s'est construite à un moment donné et qui fait désormais partie de son point de vue. Il faudrait donc parvenir à faire la part des choses entre le vécu réel, le vécu construit qui fonde un point de vue, ou un discours factice qui tient à la situation d'entretien. La subjectivité est ensuite celle de l'observateur dans le traitement de discours contradictoires et dans l'évaluation de l'importance de chaque événement. Il est donc important de toujours pouvoir se référer aux récits de chacun des acteurs, qui restent le matériau de base de l'analyse. Le suivi des processus en temps réel est une réponse à ces limites, mais il est délicat d'être accepté comme simple observateur dans des conflits aigus. Enfin, l'observateur utilise toujours une porte d'entrée pour prendre pied dans la réalité. Tel agent de développement nous indique des acteurs à rencontrer, qui nous indiquent à leur tour d'autres acteurs, etc. : on pénètre alors dans un réseau qui peut n'être qu'une partie du jeu d'acteurs. Il sera donc capital d'utiliser plusieurs portes d'entrée.

\section{Un cadre conceptuel issu des premières observations}

\section{Des itinéraires de concertation : définition et composantes}

L'analyse comparative des processus nous conduit à parler d'itinéraires de concertation. À l'image de l'itinéraire technique d'une culture, marqué par des étapes 
de croissance de la plante et différentes interventions effectuées par l'agriculteur, l'itinéraire de concertation est marqué par une progression dans le dialogue (positive ou négative), des «événements » extérieurs qui l'influencent et d'éventuelles interventions visant à favoriser son avancée, qualifiées d' "opérations ». Il est décomposable en phases qui peuvent soit se superposer pendant un certain temps, soit se succéder, suite à un événement qualifié de "charnière ». Le nombre et la nature des participants, l'objet de discussion, l'émergence d'accords, sont les éléments qui permettent de caractériser chaque étape du processus. Finalement, l'itinéraire de concertation représente le cheminement suivi par la concertation en termes de contenu comme de forme. Chaque itinéraire est unique et ne peut être assimilé à une procédure, définie dans le cas de processus formalisés comme un ensemble de règles et de formalités qui doivent être observées pour parvenir à un résultat (Candau, 1999) : dans le cas de processus formalisés, une procédure fixe parfois un cadre et une ossature que l'itinéraire respecte mais déborde.

Cet itinéraire débute avant la mise en place d'instances de concertation, lorsque son objet émerge dans l'espace public, sous la forme soit d'un conflit, soit d'une proposition individuelle ou institutionnelle. Par exemple, en rivière de Penerf, 1 'objet premier de la concertation est la qualité de l'eau de la rivière et plus précisément le respect de la règle dite de la bande des 500 mètres, qui interdit aux agriculteurs d'épandre des fertilisants organiques à moins de 500 mètres du rivage, en amont de zones conchylicoles. Or, cet objet émerge publiquement lors de la réalisation d'une étude relative à l'aménagement foncier, de façon fortuite : il existe déjà des pollutions et des conflits latents, mais la combinaison qualité de l'eau/règle de la bande des 500 mètres/conflit ostréiculteurs-agriculteurs littoraux, qui constitue l'objet premier de la concertation, n'émerge qu'à l'occasion de cette étude, qui signale l'existence d'une règle localement ignorée jusqu'alors. Cet objet évoluera ensuite au cours du processus.

Chaque processus est composé de plusieurs éléments qui le caractérisent. La préoccupation commune qui est à l'origine du processus de concertation se nomme objet de concertation. Situé au cœur des relations entre les acteurs, il est défini par plusieurs éléments. Cet objet donne lieu à des controverses, c'est-à-dire un désaccord qui peut porter soit sur l'objet dans son ensemble, soit sur une déclinaison de celui-ci. Par exemple, si l'objet est la mise en place d'un outil de gestion, une déclinaison possible est la définition de son périmètre. Cette controverse est structurée par des points de vue différents sans qu'il y ait nécessairement conflit.

La concertation prend forme sur des scènes de concertation autour desquelles s'articulent les échanges entre acteurs. Une scène de concertation est assimilable à celle d'un théâtre : elle regroupe un certain nombre d'acteurs dans une histoire dont on attend et espère le dénouement. De même que le décor d'une pièce de théâtre est amené à changer, la scène va se matérialiser en des lieux divers. L'histoire est faite d'une controverse, le dénouement sera l'accord ou le désaccord. Chacun est caractérisé par le personnage (ici le groupe social) qu'il représente, mais le scénario n'est pas écrit à l'avance et sera le fruit du dialogue. Nous distinguons la scène de l'arène telle que définie par Olivier de Sardan (2001), qui entend par ce terme qu'autour d'enjeux ou de problèmes, «les acteurs se divisent en une pluralité d'attitudes, de représentations, de normes, qui se confrontent ». L'arène n'est pas un regroupement conscient, mais un espace de confrontation entre des acteurs sociaux en interaction autour d'enjeux communs. La métaphore théâtrale conduisant à l'utilisation du terme «scène » est utilisée par Decrop et Vidal-Naquet (1998) à propos des scènes locales de risque : il y a scène s'il y a mise en scène du risque, c'est-à-dire mise en visibilité, multiplication des pièces jouées et des objets ; les acteurs peuvent passer d'une scène à une autre, voire traiter le risque dans les coulisses, à l'écart de la scène visible. L'expression "scène locale de risque » désigne ainsi «des lieux formels ou informels, où des acteurs sociaux se rassemblent en vue de gérer le risque » (Decrop et al., 2001). Un itinéraire va souvent évoluer sur plusieurs scènes et pourra, comme nous allons le voir ultérieurement, rebondir sur des scènes qui n'ont a priori rien à voir avec son objet.

Ce cadre conceptuel s'applique aussi bien à des dynamiques de concertation où l'on progresse vers un accord qu'à des concertations qui se figent, avec la cristallisation de positions opposées, où l'itinéraire est surtout celui d'un conflit. Nous nous baserons ici avant tout sur l'analyse de dynamiques où un accord se construit, l'analyse d'itinéraires de conflit étant relatée dans d'autres contributions (Pennanguer et al., 2004).

\section{Un cadre théorique : de la concertation à l'innovation}

L'analyse et la représentation des itinéraires de concertation nous ont permis de constater que tous progressent via la consolidation puis l'élargissement du réseau des participants. Au départ, ce réseau est limité aux quelques catégories d'acteurs qui acceptent le dialogue, à une échelle et autour d'un objet eux-mêmes restreints, puis le réseau s'étoffe. Certes, il arrive que l'accord se noue via une réduction drastique de ce réseau, comme nous l'avons observé dans des travaux antérieurs (Beuret, 1999), le moyen le plus rapide d'aboutir à un accord étant de marginaliser les participants qui ont des points de vue et des références différents. Mais l'accord est alors le triomphe d'un point de vue déjà établi aux dépens des autres, et une telle réduction est un échec de la concertation en tant que construction collective d'objets communs. Si l'on exclut ces cas - considérés comme des 
échecs -, la concertation progresse toujours via l'élargissement du réseau : l'implication de nouvelles catégories d'acteurs donne plus de poids à la dynamique engagée et, au-delà du dialogue, la mise en place de porte-parole, de supports $d$ 'information mutuelle, de règles de dialogue... permet de consolider le réseau qui porte la concertation et les accords qui pourront en résulter.

Nous retrouvons ici ce que nous apprend la sociologie de l'innovation (Callon et Latour, 1991; Amblard et al., 1996). S'intéressant à diverses innovations, ces auteurs constatent que ce n'est pas la qualité intrinsèque de l'innovation qui fait qu'elle va ou non s'imposer, mais le processus sur lequel elle s'appuie, et en particulier la consolidation et l'élargissement d'un réseau qui la porte. Selon ces auteurs, le réseau est un ensemble d'entités humaines ou non humaines (par exemple les huitres, qui réagissent à la dégradation du milieu), individuelles ou collectives, définies par leur rôle, leur identité, leur programme : il relie toutes les entités qui participent au problème et à la recherche de solutions. Ce réseau va se consolider par rallongement, en intégrant de nouvelles entités, jusqu'à son irréversibilisation (Callon, 1994). Ceci coïncide avec ce que nous avons observé: par exemple à Étel, les acteurs affirment que $l^{\prime}$ « on ne pourrait pas revenir en arrière », ce qui revient à dire que le réseau s'est cristallisé de façon irréversible.

Dès lors, la concertation doit être vue comme un processus de construction collective d'une innovation sociale, portée par un réseau dont la consolidation et l'élargissement déterminent le succès.

\section{L'analyse comparative d'itinéraires de concertation : résultats}

\section{Les itinéraires de concertation : structure et logique d'ensemble}

Nous nous basons ici sur l'exemple de la ria d'Étel. L'itinéraire de concertation est représenté sous la forme d'un arbre, issu d'une graine, doté d'un tronc central, puis de branches qui élargissent l'arbre jusqu'à ce qu'il donne des fruits (Fig. 1).

\section{Une représentation arborescente}

\section{La graine, ou la révélation de l'objet}

La révélation de l'objet correspond à l'événement qui fait émerger l'objet de la concertation dans le débat public. À Étel, la qualité de l'eau et les pollutions d'origine agricole sont des objets de conflits latents. Ils émergent sur la scène publique lorsqu'un agriculteur épand du lisier si proche du rivage qu'il s'enlise au bord de la rivière : pour sortir de ce mauvais pas, il y déverse son chargement, en amont de concessions ostréicoles. Un mouvement de colère explicite alors le conflit et pose l'objet dans l'espace public local. De même que la composition d'une graine détermine en grande partie la nature de la plante à laquelle elle va donner naissance, la façon dont l'objet est posé dans l'espace public et les éléments qui le composent se révèlent très déterminants pour la suite du processus. Par exemple à Étel, les agriculteurs sont directement mis en accusation, alors que certaines stations d'épuration auraient pu l'être : le binôme agriculteurs-ostréiculteurs sera au centre de tout le processus. La révélation de l'objet peut être accidentelle ou volontaire. La graine trouvera un terreau favorable si le problème a suffisamment d'acuité pour mobiliser les énergies locales.

\section{Les racines ou le réseau des participants}

Les catégories d'acteurs qui se mobilisent sont autant de « racines » qui stabilisent le processus, et ceci, qu'elles soient pour ou contre ce processus. Elles constituent le réseau des participants. Dans le cas d'Étel, ce réseau est d'abord limité aux seuls agriculteurs et ostréiculteurs, réunis à l'initiative des présidents des syndicats locaux des deux professions. Un climat de dialogue s'installe grâce à la proximité entre les deux leaders. D'autres participants vont se joindre à eux lors de phases ultérieures, notamment des élus et d'autres organisations agricoles. Ces nouvelles racines viendront consolider l'édifice en lui donnant plus de reconnaissance et en apportant des ressources humaines et financières supplémentaires.

\section{Le tronc, ou le réseau de base des participants}

Le tronc de l'arbre est composé du premier réseau de participants, réunis sur une première scène de concertation : il est alors constitué, en termes de participants et de thématique abordée, des seuls éléments qui permettent d'engager un dialogue. Ceci conduit dans un premier temps à éviter les sujets de conflits. À Étel, les leaders agricoles et ostréicoles parviennent à former un premier réseau dans un espace de concertation qualifié de « groupe professionnel local ». Pour cela, ils proposent à chacun de venir dialoguer et se (re)connaître sans aborder d'emblée le conflit existant. Des visites mutuelles, qualifiées d'échanges " paysans de la terre - paysans de la mer », permettent aux participants de mieux se connaître et de se découvrir une proximité de métier. À ce stade, la consolidation de ce premier «tronc commun » repose sur deux ressorts :

- un apprentissage commun : il s'agit d'apprendre ensemble et d'apprendre de l'autre pour se forger des références communes. À Étel, les agriculteurs et les ostréiculteurs s'aperçoivent ainsi que le terme de pollution n'a pas le même sens pour les uns 


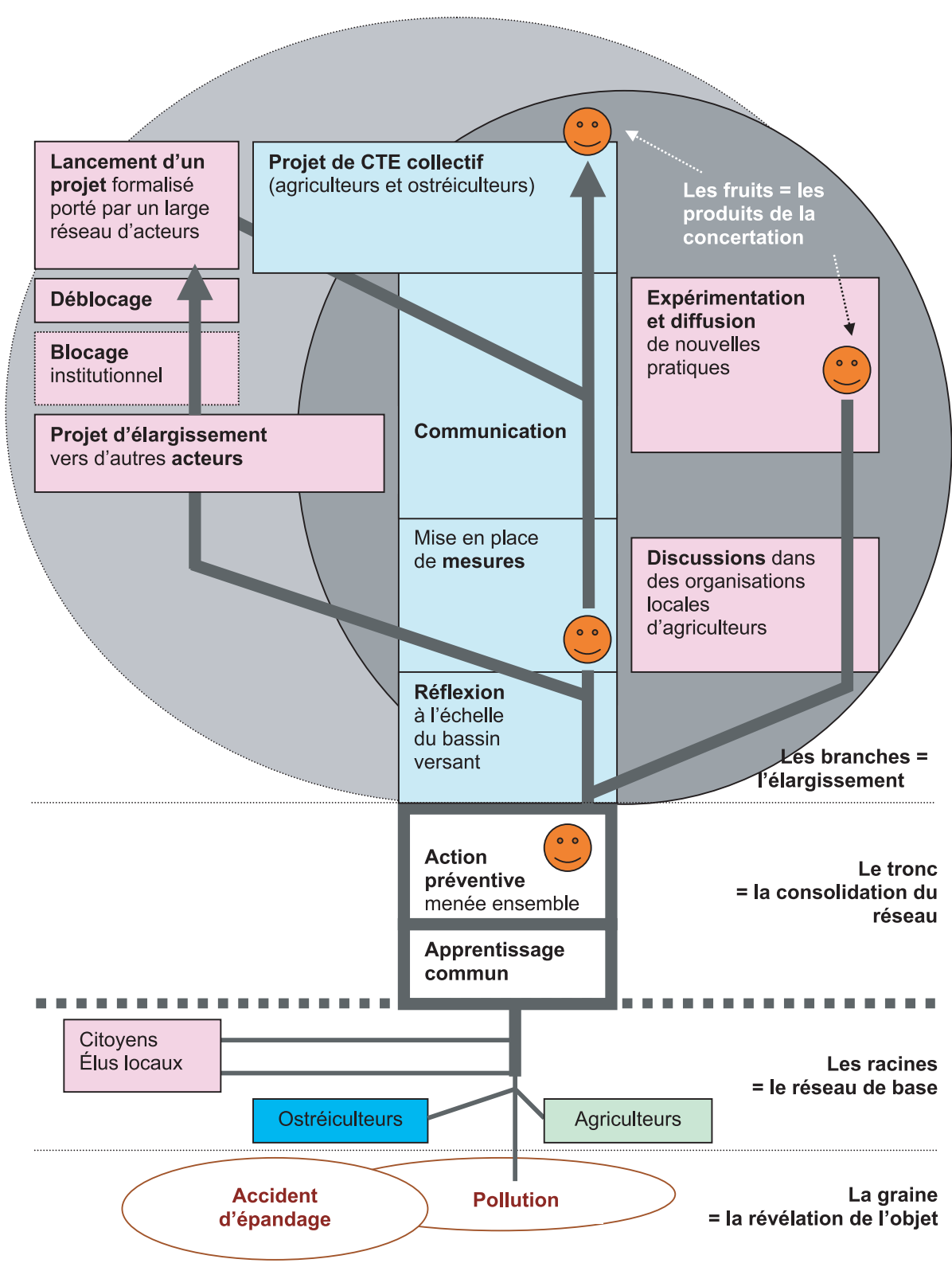

Fig. 1. La représentation d'un itinéraire de concertation.

et pour les autres : les agriculteurs pensent pollution par les nitrates (pour laquelle la profession est mise en cause publiquement), lorsque les ostréiculteurs pensent aux pollutions bactériologiques (qui leur posent problème). Ces premières discussions permettent la construction d'un savoir commun. La conduite d'une action commune, quelle que soit son efficacité, va dans le même sens ;

- l'identification de valeurs communes, d'un sens commun : pourquoi se concerter si nos intérêts divergent et si tout nous sépare? Dès lors, on cherche à ce stade, plus ou moins explicitement, à se retrouver autour de valeurs (liées au métier dans le cas d'Étel), d'un attachement au territoire, de n'importe quel élément qui révèle l'existence d'intérêts communs, et constitue alors un objet-frontière tel que défini par Star et Griesemer (1989). Selon ces auteurs, les objets-frontières sont situés à l'interface entre plusieurs mondes, mais répondent aux nécessités de chaque monde; ils sont "suffisamment flexibles pour s'adapter aux besoins et aux nécessités spécifiques des différents acteurs qui les utilisent et sont suffisamment robustes pour maintenir une identité commune » (Flichy, 2003). Ce sont des dispositifs qui permettent une articulation souple entre positions différentes, tout en fixant un cadre d'action structurant (Hert, 1999). Les valeurs communes identifiées entre des acteurs ayant des points de vue différents peuvent être considérées comme des objets-frontières. 
Lors de la constitution de ce réseau de base, l'évitement du conflit est une constante que l'on retrouve dans l'ensemble des processus étudiés. Dans un autre cas, la première chose que font ensemble des conchyliculteurs et des agriculteurs, au cœur d'un conflit, est d'aller visiter... une station d'épuration!

\section{Les branches : l'élargissement et la consolidation du réseau}

Le premier réseau de participants s'élargit vers d'autres acteurs, et le contenu des discussions pénètre alors d'autres sphères qui constituent autant de branches de l'arbre. Dans le cas d'Étel, le tronc que constitue le groupe professionnel local continue à pousser de façon linéaire, avec la mise en place de mesures agrienvironnementales, des opérations de communication qui visent à "marquer les esprits », selon les dires des acteurs, puis la préparation d'un projet de contrat territorial d'exploitation associant les agriculteurs et les ostréiculteurs, chacun devant s'engager sur des mesures favorables au territoire. Une première ramification pénètre les organisations agricoles locales, qui vont discuter de pratiques visant à préserver la qualité de l'eau, expérimenter certaines d'entre elles, puis les diffuser. Une seconde ramification élargit le processus vers les collectivités publiques locales et leurs élus et vers une échelle plus adaptée, mais qui ne pouvait être abordée d'emblée, celle du bassin versant. Ces ramifications permettent l'élargissement du réseau et sa consolidation, de nouvelles racines venant stabiliser l'édifice.

\section{Les fruits, produits de la concertation : des accords} contractuels ou conventionnels

Ce sont les résultats concrets de la concertation, en termes d'accords et d'actions concertées. Les accords peuvent prendre une forme contractuelle : ce sont des contrats et des plans de gestion, des cahiers des charges, des codes de bonnes pratiques, etc., matérialisés par un contrat écrit ou oral, une adhésion manifestée par une signature ou un engagement moral, un système de contrôle et de sanctions. Mais l'accord peut rester entièrement tacite et prendre une forme conventionnelle. Il s'agit alors d'un accord tacite autour d'une certaine discipline collective. Ces conventions (Favereau, 1989) se manifestent par des comportements identiques, conformes à des règles énoncées sous des formes telles que « je ne me permettrais jamais de... ( (Beuret, 1998). Dans les cas étudiés, il s'agit surtout de conventions d'effort, qui établissent "le niveau d'effort communément admis comme normal» (Gomez, 1994). À Étel, la plupart des agriculteurs locaux respectent désormais la loi relative à la bande des 500 mètres, alors qu'ils ne le faisaient pas avant sans pour autant être inquiétés : il s'agit donc d'un effort collectivement consenti, et, lorsque l'un d'entre eux a violé cette règle, il a reçu la visite d'une délégation composée d'agriculteurs et d'ostréiculteurs, preuve de l'existence d'un contrôle social très étroit.

L'un des fruits de la concertation est un changement durable dans les relations entre acteurs : s'étant côtoyés, il leur est plus difficile d'assumer un comportement hostile vis-à-vis des autres ou de bafouer des règles collectivement évoquées. Dans plusieurs cas étudiés, les participants disent que «les comportements ont changé », en l'absence de tout accord formel.

Tous les itinéraires de concertation que nous avons étudiés peuvent être représentés de cette façon, ce qui est très intéressant pour renvoyer une image du processus aux acteurs locaux et leur faire prendre du recul quant au chemin parcouru et à celui qui reste à parcourir. Cependant, nous verrons par la suite que l'arbre est parfois bien tourmenté, voire nébuleux...

\section{Une logique d'ensemble : de l'acceptable au souhaitable}

L'itinéraire de concertation se base toujours sur un premier «acceptable commun ». Le réseau et l'objet de la concertation, lorsqu'elle s'engage, sont toujours très restreints. Ils correspondent à un plus grand dénominateur commun issu des réponses à la question : «Avec qui et à propos de quoi j'accepte de dialoguer? » La réponse commune à cette question s'élargira au fur et à mesure du processus, condition sine qua non de l'élargissement du réseau dans des phases ultérieures. La logique d'ensemble qui dicte sa dynamique à l'itinéraire est la suivante : des participants dialoguent autour d'un «acceptable commun », puis cet «acceptable » s'élargit grâce au dialogue, jusqu'à permettre l'élargissement du réseau et/ou de l'objet. Le réseau élargi engage le dialogue sur la base d'un nouvel «acceptable » destiné à s'élargir à nouveau.

Ce processus permet de passer de l'acceptable au souhaitable. À Étel comme en rivière de Penerf, le premier réseau n'est constitué que des agriculteurs et des ostréiculteurs de l'une des communes riveraines de la rivière. C'est l'échelle à laquelle les participants acceptent le dialogue : elle est pertinente du point de vue social, mais pas en termes de gestion. Progressivement, le réseau va s'élargir à d'autres communes littorales, puis à l'échelle pertinente de gestion concertée des pollutions d'origine agricole, c'est-à-dire le bassin versant : cette échelle ne pouvait être considérée d'emblée et ne l'est qu'à la suite d'un processus itératif de stabilisation du réseau, de légitimation de ses participants, puis d'élargissement. Il en va de même en termes de catégories d'acteurs : les citoyens et les élus sont d'abord exclus de la concertation et ne seront associés qu'à la suite de la consolidation d'un premier réseau restreint aux agriculteurs et aux ostréiculteurs; ces derniers vont exploiter des proximités 
de métier pour engager des rapprochements. Le processus permet de passer de l'acceptable au souhaitable, en termes de participants, d'objet et d'échelle.

\section{Conséquences opérationnelles}

Ceci a d'importantes conséquences opérationnelles. Là où un animateur tente d'imposer d'emblée sa vision du souhaitable, donc de réunir tous les acteurs concernés pour aborder l'ensemble du problème à une échelle pertinente du point de vue environnemental, on ne peut que constater de nombreux échecs. Son rôle est de conduire la concertation en respectant la logique d'ensemble que nous venons de décrire. Cette logique est celle d'un processus d'innovation qui progresse via la consolidation et l'élargissement du réseau : l'animateur de la concertation se fait alors traducteur au sens des sociologues de l'innovation. La traduction est un mouvement qui vise à «établir un lien intelligible entre des activités hétérogènes » (Callon et Latour, 1991). Lorsque les uns parlent de produit brut à l'hectare, les autres des chemins creux de leur enfance et d'autres encore de biodiversité et de générations futures, une traduction qui vise à lier « des énoncés et des enjeux a priori incommensurables et sans commune mesure » prend tout son sens. Le traducteur rapproche, relie, assemble le réseau, et les références fournies par la sociologie de l'innovation (Amblard et al., 1996) se révèlent pertinentes pour analyser cette fonction et préciser ses contours (Beuret, à paraître).

\section{Lorsque la concertation rebondit sur plusieurs scènes}

\section{La rivière de Penerf : parce que chacun n'en fait pas le même récit}

Les récits des acteurs d'une même concertation sont souvent si différents que ces derniers semblent ne pas avoir vécu la même chose. C'est le cas en rivière de Penerf, alors que la concertation se développe au sein d'une même commune. Les récits diffèrent quant au début, aux étapes et à la fin de l'action.

- Le début de l'action et ses antécédents : ostréiculteurs et agriculteurs évoquent un premier conflit qui oppose les ostréiculteurs à un industriel, puis une étude qui révèle l'existence de la règle d'interdiction d'épandage dans la bande des 500 mètres. Une agricultrice évoque une rencontre qu'elle a organisée entre agriculteurs et ostréiculteurs. Ces événements, qui déterminent la façon dont les acteurs arrivent dans la concertation, ne sont pas cités par les autres protagonistes.

- Une première scène de concertation est mise en place par les animatrices d'un syndicat intercommunal, d'une part, et de la chambre d'agriculture, d'autre part.
Tous les participants évoquent cette scène qui disparaît prématurément : une partie des agriculteurs quitte la scène, puis survient la marée noire de l'Erika et, selon un agriculteur, «l'ennemi $\mathrm{n}^{\mathrm{o}} 1$ de l'ostréiculteur n'est plus l'agriculteur mais le pétrolier », et la concertation s'arrête.

- Alors que certains situent ici la fin de l'action, de nouvelles scènes sont citées par les autres protagonistes. C'est d'abord la Commission communale d'aménagement foncier (scène $n^{\circ} 2$ ), où ce qui a été évoqué dans la scène $n^{0} 1$ est repris en l'absence des ostréiculteurs, mais avec l'irruption d'environnementalistes. La discussion rebondit une nouvelle fois lors de la constitution des listes pour les élections municipales : le futur maire voulant intégrer des représentants de chaque profession dans sa liste, la concertation reprend sur cette nouvelle scène $\left(\right.$ scène $\left.n^{\circ} 3\right)$. Le maire situe ici la fin du processus et affirme vouloir le relancer. Pourtant, les agriculteurs comme les ostréiculteurs affirment qu'il continue dans le cadre d'un accord négocié à l'échelle départementale entre les deux professions et dont l'application suppose un accord local entre les deux catégories d'acteurs (scène $\left.\mathrm{n}^{\mathrm{o}} 4\right)$.

- Des résultats obtenus ailleurs. Enfin, les agriculteurs citent des résultats obtenus via leurs organisations professionnelles, tels que l'achat d'une composteuse et l'adoption de certaines pratiques agricoles, qui n'ont fait l'objet d'aucun contrat avec les autres acteurs, mais qui sont des acquis du processus.

Que déduire de cet imbroglio apparent? Au-delà des lieux spécifiquement voués à la concertation autour d'un objet, les choses avancent aussi via les réseaux d'appartenance de chaque acteur et sur des scènes qui n'ont rien à voir avec ces lieux. Dans le cas de Penerf, la concertation rebondit sur plusieurs scènes sans aucun lien apparent; pourtant, elles supportent la progression du processus.

\section{Comment l'itinéraire mène-t-il d'une scène à l'autre?}

Le passage d'une scène à l'autre n'a souvent rien d'ex-

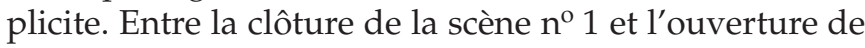
la scène $\mathrm{n}^{\mathrm{O}} 2$ se sont écoulés huit mois, durant lesquels le débat public a cessé. Il est réapparu de lui-même après ce qui n'était qu'une période de latence. Par qui et comment les termes du débat sont-ils archivés puis transmis d'une scène à l'autre?

Le lien se fait essentiellement par des personnes qui sont soit présentes dans plusieurs scènes (des agriculteurs présents dans les scènes $n^{\circ} 1$ et $n^{\circ} 2$ ), soit informées et qui portent le débat dans des scènes où elles sont présentes : les supports locaux d'information sont alors des vecteurs essentiels, de même que des personnes relais marquées par une multi-appartenance qui peut être horizontale, du local vers le local, et/ou verticale, du local vers le global. C'est, par exemple, un ostréiculteur dont les parents sont agriculteurs. Dans un premier temps, 
ses parents vont induire l'élargissement de la scène $\mathrm{n}^{0} 1$ aux ostréiculteurs, alors qu'elle ne comprenait que les agriculteurs et les élus communaux. Cet ostréiculteur joue ensuite un rôle essentiel dans la scène $\mathrm{n}^{\mathrm{o}} 3$. Il intègre une association regroupant des conchyliculteurs, des agriculteurs et des pêcheurs, qui constitue une scène de concertation externe : elle va mener des actions locales qui entrent dans la poursuite de l'itinéraire de concertation, puis dans la mise en œuvre des accords issus de la scène $\mathrm{n}^{\circ} 4$.

On observe ensuite que la concertation se déplace implicitement vers les scènes qui lui sont plus favorables, même lorsque ces scènes ne sont pas celles qui ont été conçues pour elle, en vertu d'un principe d'adaptation. Les agriculteurs lourdement mis en cause ont quitté la scène $n^{0} 1$, refusant le dialogue dans une scène qu'ils ne contrôlaient pas. Ils acceptent le même dialogue dans la scène $\mathrm{n}^{\mathrm{o}} 2$ où, selon l'un d'entre eux, "c'est nous qui avons le pouvoir ». Ils sont certes sous la pression des écologistes et des ostréiculteurs, mais cette scène leur offre certaines garanties qui manquaient à la scène $n^{0} 1$ : ceci permet la réactivation du débat, dans une scène qui n'est pas prévue à cet effet. Le débat renaît ensuite dans la scène $n^{\circ} 3$, qui lui offre une inscription dans la durée, une légitimité et une pluralité de représentations qui manquaient à la scène $n^{\circ} 2$. Ce principe d'adaptation explique aussi des périodes de latence. Elle entre en latence pour renaître lorsque les conditions lui sont à nouveau favorables.

\section{Déductions méthodologiques : la concertation ou le choc des réseaux}

Ces constats nous amènent à revenir sur la définition de la concertation : elle correspond à l'interpénétration ponctuelle ou durable de réseaux d'acteurs, sur une ou plusieurs scènes, réseaux qui vivent et évoluent en dehors d'elle. La concertation alimente ces réseaux et les fruits de la concertation sont parfois à rechercher au bout de ces réseaux, bien loin des espaces de concertation proprement dits. La rencontre entre les réseaux d'acteurs se fait sur des scènes qui n'ont parfois aucun lien formel entre elles, mais qui sont au cœur d'un champ où la problématique vit et est portée par certains acteurs. Ce champ est à la fois centré sur un espace géographique et ouvert sur des réseaux connectés à des échelles supérieures. La question posée dans la concertation vit dans ce champ, ce qui signifie qu'elle peut y être reprise à n'importe quel moment, par n'importe quel acteur et dans n'importe quel lieu, indépendamment d'une procédure ou d'un animateur donné.

En termes méthodologiques, si la concertation évolue hors de scènes spécifiquement prévues pour elle, cela signifie que l'étudier comme un dialogue ayant lieu sur une scène donnée et conduit par telle ou telle institution a peu de sens. Toute concertation sera d'abord identifiée par son objet, les acteurs qu'elle mobilise et éventuellement une scène centrale, lorsqu'elle existe. Mais, au-delà de ce "premier plan », devront être approchées d'autres scènes qualifiées de centrales ou de périphériques : dans les scènes centrales, l'objectif reste la concertation autour de cet objet, alors que, dans les scènes périphériques, l'objet n'intervient que de façon fortuite. Elles seront replacées dans le champ de la concertation, c'est-à-dire l'espace dans lequel les acteurs concernés par l'objet de la concertation sont amenés à se rencontrer fréquemment et où cet objet resurgit au gré de ces interactions. Enfin, les réseaux d'appartenance des acteurs, qu'ils soient professionnels, militants ou même confessionnels, devront être considérés : nous nous limiterons ici aux maillons de ces réseaux qui sont influencés par la concertation et qui l'influencent.

\section{Conséquences opérationnelles}

Ceci a là encore d'importantes conséquences opérationnelles. Certains animateurs chargés de mettre en place et de conduire des concertations voient leur rôle avant tout dans la mise en place d'instances réunissant l'ensemble des acteurs concernés, avec une représentation équilibrée. Le cas de Penerf montre que ce n'est parfois ni possible ni souhaitable et que le rôle de l'animateur est d'abord de faciliter et d'accompagner la concertation là où elle se développera le mieux, indépendamment de tout a priori.

L'animateur doit ensuite mettre en place une veille permanente. Il devra s'intéresser à ce qui se passe entre chacune de ses interventions, non seulement sur d'autres scènes, mais aussi dans les réseaux d'appartenance de chaque partie en présence.

Enfin, force est de s'interroger sur le sens donné à l'interruption d'une concertation : cet arrêt est souvent vécu comme un échec, alors que le cas de la scène $\mathrm{n}^{\mathrm{o}} 1$ montre l'inverse. Selon un agriculteur, «c'est reculer pour mieux sauter ", et le maire parle d'une " panne sèche, peut-être parce que cela avait besoin de mijoter ». Les termes finaux du débat sur la scène $\mathrm{n}^{\circ} 1$ seront les termes initiaux de ce débat sur la scène $n^{\circ} 2$ : cet échec n'est pas une perte, mais un «archivage pour réactivation ultérieure », dans des conditions plus favorables. Ceci confirme la nécessité d'un pilotage souple, l'animateur devant laisser le temps aux acteurs de vivre leur "panne sèche ». Un blocage n'est un réel échec que lorsqu'il intervient sur une scène qui offre déjà des conditions optimales pour la concertation ou lorsque la concertation n'a pas suffisamment d'énergie propre pour rebondir. Cette énergie semble dépendre à la fois du niveau de conflit suscité par l'objet et de l'importance de l'enjeu perçu par les acteurs autour de cet objet, termes qui peuvent être indépendants. 


\section{La morphologie de la concertation : trois types d'itinéraires}

Si l'animateur doit accompagner la concertation là où elle trouve les conditions les plus favorables à son développement, mieux vaut qu'il sache à quoi s'attendre ! Or, l'analyse comparative des itinéraires de concertation de l'échantillon CONCERT révèle l'existence de trois morphotypes d'itinéraires, représentés dans la figure 2.

\section{Des itinéraires linéaires monocentrés}

Le cas d'Étel est caractéristique des itinéraires linéaires monocentrés. Il existe un espace de concertation central, à partir duquel la concertation se développe et va pénétrer des réseaux et des sphères différentes. Le processus est :

- linéaire : l'arbre pousse bien droit et, s'il existe différentes scènes de concertation, elles sont clairement liées à la scène centrale par des acteurs, des actions, des organisations ;

- continu : même lorsque des désaccords existent, le dialogue continue dans la scène centrale qui supporte l'ensemble de l'édifice. À Étel, les temps de crise n'ont jamais mis en cause la permanence de l'espace central de concertation ;

- monocentré : l'ensemble des actions menées reste lié $\mathrm{au}$ «tronc commun ».

On trouve ce type de processus soit hors conflits, soit lorsque le conflit reste jugulé par les acteurs locaux ou un leader jouissant d'une forte autorité.

\section{Des itinéraires séquentiels polycentrés}

On trouve des itinéraires séquentiels polycentrés dans des cas marqués par des conflits aigus autour d'objets porteurs d'enjeux importants. L'itinéraire est :

- séquentiel : des séquences de concertation se succèdent sur des scènes apparemment sans rapport les unes avec les autres. Le cas de Penerf en est l'exemple type : l'objet de la concertation erre entre des séquences successives ou parallèles de concertation, au sein d'un champ de concertation ;

- discontinu : la dissolution d'une scène peut être suivie d'une période de latence pendant laquelle les acquis antérieurs du dialogue restent ancrés dans les esprits pour constituer le point de départ de dialogues futurs. Ils entrent dans la "mémoire » du territoire (Pennanguer, 2005);

- polycentré : le tronc commun se dissout totalement ou partiellement au profit d'autres branches, puis le processus passe d'une scène à l'autre en l'absence de toute scène centrale.
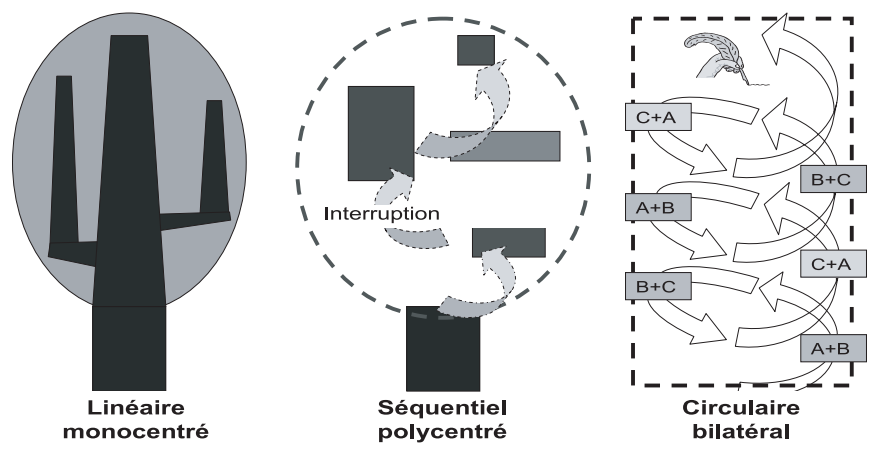

Fig. 2. Trois morphotypes d'itinéraires de concertation.

Nous avons ainsi, dans le cas de Penerf, quatre scènes que rien ne relie formellement, ni une institution ni même leurs objectifs. L'itinéraire n'est ni organisé ni maîtrisé. Les acquis d'une séquence de concertation, qu'il s'agisse d'accords ou de désaccords, sont mis en mémoire. Une énergie s'accumule, d'autant plus forte que le conflit est violent et/ou les enjeux importants : elle permet à la concertation de refaire surface dès que l'occasion s'en présente, au sein du champ de concertation.

\section{Des itinéraires circulaires bilatéraux}

On trouve enfin des itinéraires circulaires qui progressent via des contacts bilatéraux. Soit il existe des scènes qui associent des acteurs deux à deux, sans aucun contact multilatéral, soit un ou plusieurs acteurs font circuler l'information et progresser la réflexion en allant d'une catégorie d'acteurs à une autre, sans que jamais ceux-ci ne se rencontrent. Nous restons dans une logique de concertation si l'on considère que les idées progressent et se construisent par la confrontation des points de vue et des propositions de chaque acteur, même si elle est ici orchestrée par un tiers.

L'échantillon CONCERT comprend ainsi une action collective menée autour du paysage dans une commune périurbaine, avec trois acteurs : une association de résidents, la municipalité et une CUMA ${ }^{2}$ qui regroupe l'ensemble des agriculteurs de la commune. Durant huit ans, l'association va mener des actions en concertation avec la municipalité autour du paysage en milieu urbain, avec une journée annuelle de l'arbre, la mobilisation de la population pour des opérations de plantation, la signature d'une charte pour le paysage. Parallèlement, une concertation bilatérale a lieu entre la municipalité et la CUMA au sein d'une commission extramunicipale agricultureenvironnement, avec pour produit des actions relatives à l'agriculture et la qualité de l'eau. À ces concertations bilatérales parallèles succède une concertation entre l'association et la CUMA, qui se retrouvent autour d'un projet intitulé « Réhabiliter ensemble la trame bocagère ». On observe donc une progression dans une gestion

\footnotetext{
${ }^{2}$ Coopérative d'utilisation de matériel agricole.
} 
concertée des espaces urbains et agricoles via des contacts bilatéraux qui s'alimentent les uns les autres : la circulation de l'information et la construction collective d'idées, de règles et d'actions ne se fait qu'à travers ce type de contacts.

\section{Conséquences opérationnelles}

Tout le monde préférerait la transparence et la continuité d'une concertation linéaire au désordre apparent qui règne dans les autres types de processus. Des itinéraires circulaires peuvent être mis en place volontairement par un acteur qui veut garder une mainmise totale sur l'action collective : il se pose en nœud de réseau incontournable, sans parfois que cela ne soit justifié. Mais, en général, les itinéraires séquentiels ou circulaires émergent par défaut, lorsque des blocages se manifestent. Il s'agit soit d'un blocage du dialogue sur une scène donnée, soit des réticences de certains acteurs à entrer dans un dialogue direct, car ils ne se reconnaissent mutuellement aucune légitimité ou ne peuvent pas se voir, au sens propre comme au sens figuré ! Il apparaît donc que la concertation s'oriente par elle-même vers des formes, des scènes, des modalités d'échanges qui lui sont favorables : nous retrouvons ici le principe d'adaptation. Ceci confirme la nécessité, pour un éventuel animateur, d'accompagner ce mouvement plutôt que de vouloir confiner la concertation dans des instances recommandées par tel financeur, telle procédure ou tel « expert».

\section{Conclusion}

L'analyse comparative d'itinéraires de concertation montre la nécessité de renoncer à observer la concertation comme un processus, voire une procédure, qui réunit des acteurs donnés dans une ou plusieurs instances. On s'intéressera aux scènes investies de fait par le processus de concertation au sein d'un champ beaucoup plus large. Pour nous, la concertation est définie comme un processus composé de multiples interactions entre plusieurs réseaux d'acteurs, dont certaines ont lieu dans des espaces spécifiquement mis en place à cet effet, alors que d'autres se déroulent sur d'autres scènes, au sein d'un champ d'interactions. Lorsque la concertation se heurte à des blocages, elle s'adapte par elle-même pour trouver dans ce champ les scènes les plus favorables à sa poursuite. Elle peut entrer en latence pour ne réémerger que lorsque les conditions sont réunies en termes de mobilisation des acteurs et d'existence de scènes acceptables par les parties en présence. En termes opérationnels, ceci doit conduire les animateurs de ces processus à accompagner la concertation là où elle va plutôt que lui imposer une forme et des lieux, puis à accepter qu'elle s'éteigne lorsqu'elle en a besoin pour mieux renaître ailleurs. Conduire la concertation, ce n'est pas lui tracer un chemin, mais l'accompagner tout au long d'un itinéraire dont nul ne peut connaître les contours et qui se dessine en cours d'action.

\section{Références}

Amblard, H., Bernoux, P., Herreros, G., Livian, Y.-F., 1996. Les Nouvelles Approches sociologiques des organisations, Paris, Le Seuil.

Barouch, G., 1989. La Décision en miettes : systèmes de pensée et d'action à l'œuvre dans la gestion des milieux naturels, Paris, L'Harmattan.

Beuret, J.-E., 1998. Agriculture et qualité de l'espace rural : coordinations, conventions, médiations. Thèse de doctorat en économie de l'agriculture et des ressources, ENSAR, Rennes.

Beuret, J.-E., 1999. Petits arrangements entre acteurs... Les voies d'une gestion concertée de l'espace rural, Natures Sciences Sociétés, 7, 1, 21-30.

Beuret, J.-E., à paraître. La Conduite de la concertation pour la gestion de l'environnement et le partage des ressources, Paris, L'Harmattan.

Beuret, J.-E., Tréhet, C., 2001. Pour la gestion concertée de l'espace rural : appuyer des médiations territoriales, Le Courrier de l'environnement de l'INRA, 43, 25-40.

Callon, M., 1994. Réseaux technico-économiques et irréversibilités, in Boyer, R., Chavance, B., Godard, O. (Eds), Les Figures de l'irréversibilité en économie, Paris, EHESS, 195-230.

Callon, M., Latour, B. (Eds), 1991. La Science telle qu'elle se fait : anthologie de la sociologie des sciences de langue anglaise, Paris, La Découverte.

Candau, J., 1999. Usage du concept d'espace public pour une lecture critique des processus de concertation : le cas des OLAE en Aquitaine, Économie rurale, 252, 9-15.

Decrop, G., Vidal-Naquet, P.A., 1998. Les scènes locales de risque, in CNRS, Actes de la dixième séance du programme Risques collectifs et situations de crise, à l'École nationale supérieure des mines de Paris, 19 mars 1998, Grenoble, CNRS, 15-49.

Decrop, G., Dourlens, C., Vidal-Naquet, P.A., 2001. L'opacité des scènes locales, in Boyer, M., Herzlich, G., Maresca, B. (Eds), L'Environnement, question sociale: dix ans de recherches pour le ministère de l'Environnement, Paris, Odile Jacob, 217-223.

Favereau, O., 1989. Marchés internes, marchés externes, Revue économique, 2, 273-328.

Flichy, P., 2003. Technologies, imaginaires, pratiques, (Working Paper), École thématique du CNRS Technologies de l'information et de la communication et structuration des collectifs, Carry-le-Rouet, 7-12 septembre.

Galléty, J.-C., Rousset, M. (Eds), 2000. La Concertation en aménagement : éléments méthodologiques, Lyon, CERTU.

Gomez, P.-Y., 1994. Qualité et théorie des conventions, Paris, Economica.

Habermas, J., 1978. L'Espace public [1962], Paris, Payot.

Hert, P., 1999. Internet comme dispositif hétérotopique, Hermès, 25, 93-107.

Ladrière, P., 1992. Espace public et démocratie, in Cottereau, A., Ladrière, P. (Eds), Pouvoir et légitimité : figures de l'espace public, Paris, EHESS, 19-44.

Lamoureux, J., 1996. La Concertation : perspectives théoriques sous l'angle du néocorporatisme, Les Cahiers du CRISES, 9607.

Latour, B. (Ed.), 1992. Ces réseaux que la raison ignore, Paris, L'Harmattan. 
Mermet, L., 1992. Stratégies pour la gestion de l'environnement : la nature comme jeu de société ?, Paris, L'Harmattan.

Mermet, L., 1998. Place et conduite de la négociation dans les processus de décision complexes : l'exemple d'un conflit d'environnement, in Faure, G.-O., Mermet, L., Touzard, H., Dupont, C. (Eds), 1998. La Négociation : situations et problématiques, Paris, Nathan, 139-172.

Olivier de Sardan, J.-P., 2001. Analyse anthropologique des conflits et groupes stratégiques, in IRMC, Corpus, sources et archives. Actes des journées d'études EHESS-IRMC «Sciences sociales : configuration en reconstruction », Tunis, 29-30 janvier 1999, Tunis, IRMC, 63-81.

Reçu le 29 mars 2004. Accepté le 30 juin 2005.
Pennanguer, S., 2005. Incertitude et concertation dans la gestion de la zone côtière. Thèse de doctorat en halieutique, ENSAR, Rennes.

Pennanguer, S., Beuret, J.-E., Tartarin, F., Sabourin, A., 2004. Se confronter pour construire? Itinéraire d'un conflit en mer d'Iroise, communication au colloque Conflits d'usage et de voisinage, Paris, 11-12 octobre.

Star, S., Griesemer, J., 1989. Institutional Ecology, Translations and Boundary Objects: Amateurs and Professionals in Berkeley's Museum of Vertebrate Zoology, Social Studies of Science, 19, 387-420.

To access this journal online: www.edpsciences.org 